\title{
Generic study on the design and operation of high power targets
}

\author{
A. Ahmad and C. Booth \\ Department of Physics and Astronomy, University of Sheffield, Sheffield S3 7RH, United Kingdom \\ D. Jenkins \\ ISIS, Rutherford Appleton Laboratory, Chilton, Didcot, Oxfordshire OX11 OQX, United Kingdom
}

T. R. Edgecock

Particle Physics Department, Rutherford Appleton Laboratory, Chilton, Didcot, Oxfordshire OX11 OQX, United Kingdom

(Received 30 September 2013; published 5 February 2014)

\begin{abstract}
With the move towards beam power in the range of 1-10 MW, a thorough understanding of the response of target materials and auxiliary systems to high power densities and intense radiation fields is required. This paper provides insight into three major aspects related to the design and operation of high power solid targets: thermal stresses, coolant performance, and radiation damage. Where appropriate, a figure-of-merit approach is followed to facilitate the comparison between different target or coolant candidates. The section on radiation damage reports total and spatial variations of displacement-per-atom and helium production levels in different target materials.
\end{abstract}

DOI: 10.1103/PhysRevSTAB.17.024701

PACS numbers: $29.25 . \mathrm{Dz}$

\section{INTRODUCTION}

Targets capable of reliable operation with high power particle beams (in the range of 1-10 MW) are a crucial component of many future accelerator facilities. There are many factors limiting their performance, including energy deposition by the beam, cooling, activation, and radiation damage. In addition, target systems and components must be operated, maintained, and replaced in a safe manner in an extremely challenging and intense radiation environment. As a result, the target is the limiting factor in the performance of a number of future facilities. Further, target and target station design, construction, and operation are specialized areas and significant improvements in performance are only achieved by the application of appropriate experience and skills.

In this paper, we identify the major challenges of high power target operation and propose measures to predict certain target behaviors under different conditions. The work presented in this paper focuses on three major issues: thermal stresses, radiation damage, and coolant performance. Because of their paramount effect on target performance, coolant thermal and neutronic effects were studied and considered part of the target system. Where appropriate, a figure-of-merit approach was followed and consequently, certain performance indicators were represented by a formula that may be generalized and applied to

Published by the American Physical Society under the terms of the Creative Commons Attribution 3.0 License. Further distribution of this work must maintain attribution to the author $(s)$ and the published article's title, journal citation, and DOI. any proposals of target designs. In some sections, where specific target designs were needed for reference, we referred to the ISIS Target Station 1 (TS1) target design. However, even in discussion of specific cases, we attempt to draw general conclusions that can be utilized in different target configurations. This work focuses only on solid targets, as they offer a simpler engineering design and manufacturing process, yet they are expected to face major challenges upon the shift to higher beam powers. The principal advantage of solid targets, however, is the fact that the radioactive isotopes produced in the target during and after the spallation process are confined to the target structure.

Four solid target materials were studied: graphite and beryllium as candidate materials for high power neutrino target $[1,2]$, and tungsten and tantalum as candidate materials for spallation targets. Inconel alloy was included as a beam window material. Inconel is very resistant to corrosion and is well suited for extreme environments [3]. Table I lists proposed future high power solid target systems and their corresponding target material and beam specifications.

\section{THERMAL STRESSES}

Induced thermal stresses in target components pose a major challenge particularly in facilities that rely on pulsed beam dynamics or continuous beam with poor reliability that causes frequent beam interruptions. Time-varying energy deposition in targets leads to nonuniform, timedependent heating of the target. This results in different rates of thermal expansion across the target, and thus peak 
TABLE I. Future high power targets [4].

\begin{tabular}{|c|c|c|c|c|c|}
\hline Facility & Target material & $\begin{array}{l}\text { Beam energy } \\
(\mathrm{GeV})\end{array}$ & $\begin{array}{l}\text { Beam power } \\
\text { (MW) }\end{array}$ & $\begin{array}{c}\text { Pulse temperature } \\
\text { rise }\left({ }^{\circ} \mathrm{C}\right)\end{array}$ & Expected \\
\hline ESS & W & 2.5 & 5 & 137 & 2019 \\
\hline SNS-STS & $\mathrm{W} / \mathrm{Ta}$ & 1.3 & 5 & $\cdots$ & $\ldots$ \\
\hline LBNE & Graphite & 120 & 0.7 & 166 & 2023 \\
\hline LBNE (upgrade) & Graphite & 120 & 2.3 & 552 & 2030 \\
\hline J-PARC-T2K (upgrade) & Graphite & 50 & 4 & $\cdots$ & $\cdots$ \\
\hline Project-X & Graphite & 3 & $1+$ & $\cdots$ & 2024 \\
\hline
\end{tabular}

stress values are regularly reached. Because the duration of a beam pulse is generally short ( $\sim 100 \mathrm{~ns}$ in ISIS TS1), the rate of heat deposition is faster than the rate of heat removal. This results in a sharp temperature rise in the target, and is commonly known as "thermal shock." It should be noted, however, that the rate at which heat is conducted through the target material during a beam pulse is less than the speed of sound. A major consequence of this repeated pattern of varying thermal stresses is the failure of a target component (such as the beam window) or the target itself, under thermal cyclic fatigue. High power beams are expected to amplify the issue of thermal cyclic fatigue as stress peaks in the target are expected to be higher.

The formation and effects of thermal stresses in high power targets have been studied extensively over the past few years [5-8]. With many target materials proposed, it is essential to be able to draw quick comparisons of the different merits of these materials, in order to facilitate the engineering design and material selection process. Rather than studying the thermal response of each target material to thermal stress separately, a figure-of-merit approach compares thermal resistances as a function of various parameters, such as temperature. As we show later, this approach can be extended to other performance-related

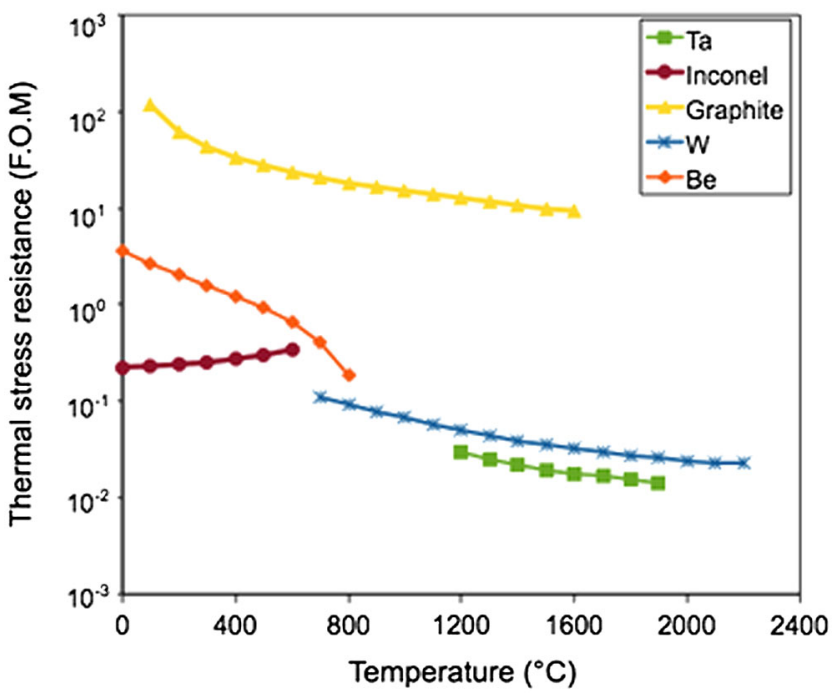

FIG. 1 (color online). Thermal stress resistance figure of merit in different target materials at different operating temperatures. issues. Figure 1 shows the variation of thermal stress resistance in different target materials with operating temperature. The melting point of tantalum, tungsten, inconel, beryllium, and graphite (sublimation) are $3020,3420,1400,1285$, and $3650{ }^{\circ} \mathrm{C}$, respectively. Graphite targets require relatively higher operating temperatures to avoid the accumulation of internal energy. Intense neutron irradiation produces defects within the graphite lattice which would increase the internal energy. At low temperatures, the energy excess is stored and might, if it were released, cause a sharp rise in the target temperature [9].

Solid targets' resistance to dynamic thermal stress depends on two parameters: the actual temperature rise in the target due heat deposition and the maximum allowable temperature for a certain target material and geometry. The temperature rise in the target due to heat deposition depends on the energy of the incident beam and the density of the target material. Charged particles of the incident beam (protons) lose part of their energy by electronic ionization upon passing through matter; this loss can be calculated using the relativistic form of the Bethe-Bloch formula [10]. Besides the ionization loss, energetic protons also undergo nuclear interactions. For beam energy higher than $100 \mathrm{MeV}$, energy loss due to nuclear interactions is dominant. Both the electronic and nuclear energy losses in the target are proportional to the density of the target material.

For a flat plate target configuration, the maximum allowable temperature difference $\left(\Delta T_{\max }\right)$ can be calculated using

$$
\Delta T_{\max }=\frac{S(1-\nu)}{Y \alpha}
$$

where $S$ is yield strength, $\nu$ is Poisson's ratio, $Y$ is Young's modulus, and $\alpha$ is the coefficient of thermal expansion.

The actual temperature rise in the target due to heat deposition created by electronic and nuclear interactions $\left(\Delta T_{\text {rise }}\right.$ ) was found to be proportional to $\rho / C_{p}$ [11], where $\rho$ is density and $C_{p}$ is heat capacity.

Therefore, the ratio between the maximum allowable temperature to the actual temperature rise in the target can be defined as a thermal stress resistance figure of merit. 
Because dynamic (time-dependent) stresses are dominant over static stresses in pulsed beam operation, the thermal diffusivity term $\frac{k}{\rho C_{p}}$ should be included to account for the effects of transient heat flow [12]. Thus, the proposed figure of merit $\left(\right.$ F.O.M $\left.{ }_{s}\right)$ used to obtain the results in Fig. 1 can be written as

$$
\text { F.O.M } \mathrm{M}_{s}=\frac{S(1-\nu) k}{Y \alpha \rho^{2}}
$$

where $k$ is thermal conductivity.

A few major observations can be made using Fig. 1. First, due to the lack of available data for some of the parameters in Eq. (2), the figure of merit was only calculated for the region where data was available. Thus, Fig. 1 can be used to highlight what material data is needed and at what temperature. For example, the thermal resistance figure of merit of tantalum was calculated for temperatures higher than $1200{ }^{\circ} \mathrm{C}$, because there was no available data for the yield strength below $1200{ }^{\circ} \mathrm{C}$. Second, in general, thermal stress resistance decreased as temperature increased. This was due to the effect of a reduction in yield strength and an increase in the coefficient of thermal expansion when temperature increased. Inconel alloy, however, seemed to retain its thermal stress resistance for temperatures in the range of $0-600{ }^{\circ} \mathrm{C}$. In fact, its figure of merit slightly increased with increased temperature. The reason for this was the steep drop in its Young's modulus compared to that of other materials. Correlations of the materials' properties with temperature were taken from the ITER materials properties handbook [13]. Third, graphite seemed to have a significantly better resistance to thermal stresses due to its low coefficient of thermal expansion. Compared to tungsten, tantalum seems to have a lower resistance to thermal stresses because of its low yield strength at high temperatures. This is in agreement with previous comparative studies of tungsten and tantalum targets reported in $[6,7]$. Beryllium was found to have the highest rate of thermal resistance decrease over the temperature range, where data was available. This was due to a relatively higher coefficient of thermal expansion.

\section{COOLANT PERFORMANCE}

Energy deposition in the target depends on beam energy, beam profile, and target geometry [14]. Cooling systems are designed to evacuate the heat produced in the target due to beam impact, plus any heat produced as a result of the decay process of radioactive target nuclei. The ideal coolant offers excellent heat transfer and has minimal neutron absorption. This section studies both of these merits for five coolant candidates: water, heavy water, helium, air, and carbon dioxide. Gaseous coolants were studied at different pressures.

\section{A. Coolant thermal performance}

Due to high energy deposition in high power systems, the cooling of target components is expected to be of a forced convection mode, through which the coolant picks up the heat when it comes into contact with the target cladding, and carries it away to the heat exchangers. The efficiency of forced convection cooling depends on the flow rate, the flow cross section, and the physical properties of the coolant and target material. Both target and coolant materials must be chosen to tolerate the maximum possible temperature.

In nuclear reactors, a conventional approach is used to compare the thermodynamic and heat transfer characteristics of different coolants. This approach is based on a figure-of-merit analysis that has been proposed by Hewitt [15], and can be defined for the lowest pumping power required for given heat flux, mass flow rate, and temperature rise in the coolant. This figure-of-merit approach can be used for the same purpose in high power target systems.

The pressure drop in a single coolant channel in the target is given by

$$
\Delta p=\frac{1}{\rho}\left(\frac{\dot{m}}{A}\right)^{2} \frac{2 f L}{D}
$$

where $\rho$ is density, $\dot{m}$ is mass flow rate, $A$ is flow cross section, $f$ is friction factor, $L$ is the channel's length, and $D$ is hydraulic diameter. On the other hand, the pumping power $P$ can be written as

$$
P=\Delta p \dot{V}=\Delta p \frac{\dot{m}}{\rho}
$$

Therefore

$$
P=\frac{1}{\rho^{2}}\left(\frac{\dot{m}^{3}}{A^{2}}\right) \frac{2 f L}{D} .
$$

The heat transferred to the coolant is given by

$$
\dot{q}=\dot{m} C_{p} \Delta T
$$

where $\dot{q}$ is heat flux, $C_{p}$ is heat capacity, and $\Delta T$ is temperature rise.

Thus,

$$
P=\frac{1}{\rho^{2} A^{2}}\left(\frac{\dot{q}^{3}}{C_{p}^{3} \Delta T^{3}}\right) \frac{2 f L}{D} .
$$

The coolant-target friction factor $(f)$ is proportional to $\operatorname{Re}^{-0.2}$, where $\operatorname{Re}$ is Reynolds number and $\operatorname{Re}^{-0.2}$ can be written as 


$$
\operatorname{Re}^{-0.2}=\left(\frac{\dot{m} D}{A \mu}\right)^{-0.2}=\left(\frac{\dot{q} D}{A C_{p} \Delta T \mu}\right)^{-0.2}
$$

where $\mu$ is dynamic viscosity.

Thus,

$$
P \propto \frac{2 L}{A^{1.8} D^{1.2}}\left(\frac{\dot{q}^{2.8}}{\Delta T^{2.8}}\right) \frac{\mu^{0.2}}{C_{p}^{2.8} \rho^{2}}
$$

For fixed $L, A, D, \dot{q}$, and $\Delta T, P$ is minimum when the (F.O.M ${ }_{c}$ ) in Eq. (10) is maximum. Therefore F.O.M ${ }_{c}$ can be written as

$$
\text { F.O.M } \mathrm{M}_{c}=\frac{C_{p}^{2.8} \rho^{2}}{\mu^{0.2}}
$$

Variations of the F.O.M ${ }_{c}$ with operating temperature are shown in Fig. 2. Both water and heavy water were considered only at atmospheric pressure, and up to $100{ }^{\circ} \mathrm{C}$. Gaseous coolants were studied up to $300{ }^{\circ} \mathrm{C}$. As illustrated in Fig. 2, temperature generally had no significant effect on the relative figure of merit of each of the candidate coolants. Both water and heavy water had far superior thermohydraulics than the other coolants considered. The difference between water and the highest value for the carbon dioxide coolant at 20 bars of pressure was 4 orders of magnitude. However, a major concern of both water and heavy water coolants is their low boiling point. In the case of a sudden power spike, loss of coolant can cause serious damage to the target equipments. Moreover, future target designs may choose to go for a gaseous coolant rather than water, because of engineering or neutronic reasons; thus, the study of other potential coolant candidates is crucial. At 1 bar of pressure, helium had slightly

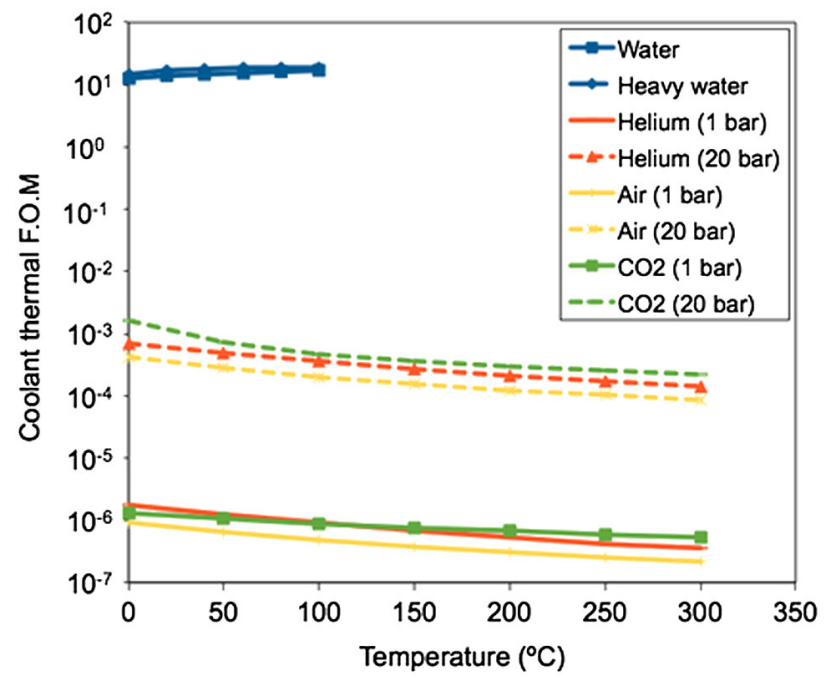

FIG. 2 (color online). Thermohydraulic figure of merit for different target coolants at different operating temperatures and pressures.

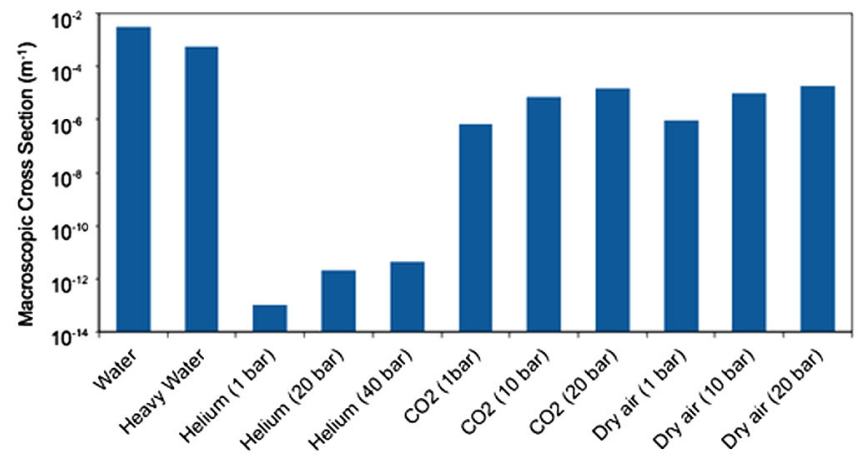

FIG. 3 (color online). Neutron absorption in different target coolants at different operating conditions.

better F.O.M $\mathrm{M}_{c}$ than that of carbon dioxide up to $100{ }^{\circ} \mathrm{C}$, and both helium and carbon dioxide were 2 and 3 times better than air, respectively. At 20 bars, the F.O.M $\mathrm{M}_{c}$ of carbon dioxide was approximately double that of helium, and 1 order of magnitude better than air.

\section{B. Coolant neutronic performance}

Besides being thermally efficient, target coolants should not affect the desired physics of the facility. In other words, the coolant should not absorb too many neutrons (or any other secondary particle of interest) and alter the required energy spectrum. Figure 3 shows neutron absorption in the target coolants. Neutrons that leak out of a target have a spallation energy spectrum that can be divided into different energy groups. A weighting factor $\left(w_{i}\right)$ was assigned to each group by dividing the number of neutrons that leaked out of the target in that energy group by the total number of neutrons that leaked out of the target. The "effective" absorption microscopic cross section $(\sigma)$ can be written as

$$
\sigma(A)=\sum_{i=1}^{n} \sigma_{i}(A) \times w_{i}(A),
$$

where $i$ represents the $i$ th energy group and $\sigma_{i}(A)$ is the microscopic cross section of element $A$ in group $i$. The four energy groups used in this case and their corresponding weighting factors are listed in Table II. The upper energy limit was set by the proton beam energy used to induce spallation in the target which was $800 \mathrm{MeV}$ (similar to the

TABLE II. Spallation spectrum energy groups and their corresponding weighting factors.

\begin{tabular}{lcc}
\hline \hline$i$ & Energy group $(i)$ & $w(i)$ \\
\hline 1 & {$[1 \mathrm{eV}, 300 \mathrm{eV}]$} & $3.52 \times 10^{-5}$ \\
2 & {$[300 \mathrm{eV}, 1 \mathrm{MeV}]$} & $5.53 \times 10^{-1}$ \\
3 & {$[1 \mathrm{MeV}, 20 \mathrm{MeV}]$} & $3.88 \times 10^{-1}$ \\
4 & {$[20 \mathrm{MeV}, 800 \mathrm{MeV}]$} & $5.93 \times 10^{-2}$ \\
\hline \hline
\end{tabular}


beam energy of the ISIS facility). The microscopic cross section of each element in the different coolants was found for every energy group using the Evaluated Nuclear Data File online database [16].

The effective absorption macroscopic cross section $\left(\sum\right)$ of a compound $A_{a} B_{b}$ was then found using

$$
\begin{aligned}
\sum\left(A_{a} B_{b}\right) & =\sum\left(A_{a}\right)+\sum\left(B_{b}\right) \\
& =\sigma(A) \rho^{\prime}(A)+\sigma(B) \rho^{\prime}(B),
\end{aligned}
$$

where $\sigma$ is the effective microscopic cross section found using Eq. (11) and $\rho^{\prime}$ is the atomic density.

Figure 3 shows that water had the highest neutron absorption. Heavy water performed slightly better, with an order of magnitude less than that of water. Helium offered the lowest neutron absorption level, with an effective macroscopic cross section in the order of $10^{-12} \mathrm{~m}^{-1}$ at 40 bars of pressure. The neutron absorption levels in carbon dioxide and dry air were very similar. It should be noted that, although water had the highest neutron absorption level, it contributed to the moderation of fast neutrons. Depending on the facility and physics required, this may or may not be beneficial.

\section{RADIATION DAMAGE}

In high power targets, radiation damage is a result of both displacement damage of lattice atoms and transmutation. Displacement damage of lattice atoms is mainly caused by collisions with incident protons, fast spallation neutrons, or recoil energy when atoms emit particles. The displaced atom moves inside the lattice until it loses all its energy creating many vacancies and interstitials. These defects in the lattice may segregate to form significant defect clusters [17]. Although the effects of radiation damage on material properties have been studied extensively in fission reactors, the energetic and diverse nature of spallation radiation fields leads to new challenges in target materials. Spallation seems to be a much harsher radiation environment than fission, and thus any extrapolation of materials' data from fission reactors is questionable.

The issue of radiation damage in high power targets is of high importance. The main challenge arises from the complexity of the different mechanisms by which radiation damage occurs, and the effects of operating conditions such as temperature on these mechanisms. Moreover, the response to radiation damage differs from one material to another. While numbers of displacement-per-atom (DPA) and gas production levels in targets can be computed using currently available Monte Carlo codes such as MCNPX or FLUKA, the real meaning of these numbers in terms of predicting effects of radiation damage is still unclear. Consequently, in this section we studied DPA and production levels of helium in target material as a conventional way of reporting a target's response to radiation damage, but without drawing conclusions about the effects of these numbers on the mechanical integrity of structural and target materials.

Although the analysis presented in this section avoids discussing the effects of radiation damage on the mechanical properties of different target materials, some experimental data exist in the literature for certain materials and at specific operating conditions. Table III lists some of the changes in the mechanical properties of graphite and beryllium that can be used as reference comparative values to the DPA and helium production levels reported in this paper. Radiation damage in graphite and beryllium targets is of particular concern because of their mechanical properties' sensitivity to proton and neutron irradiation.

The mechanical properties of graphite and beryllium targets change substantially under high energy proton and neutron irradiation. Graphite seems to acquire a negative volumetric change when irradiated. The dimensional change in irradiated graphite is anisotropic. Lattice axes $a$ and $c$ have

\begin{tabular}{|c|c|c|c|c|}
\hline Material & Conditions & Property & Value & Reference \\
\hline Beryllium & $\begin{array}{l}\text { Temperature: } 550{ }^{\circ} \mathrm{C} \\
\text { He level: } 5000 \mathrm{appm}\end{array}$ & Volume change & $0.5 \%$ & [18] \\
\hline Beryllium (LBNE) & $\begin{array}{l}\text { Temperature: } 200^{\circ} \mathrm{C} \\
\text { He level: } 1330 \mathrm{appm}\end{array}$ & Volume change & $\leq 0.5 \% /$ year & [19] \\
\hline Beryllium & $\begin{array}{c}\text { Temperature: }<230^{\circ} \mathrm{C} \\
\text { Neutron fluence: } 1.5 \times 10^{21} \mathrm{n} / \mathrm{cm}^{2} \\
\text { Neutron energy: }>1 \mathrm{MeV}\end{array}$ & Fracture toughness & $\begin{array}{c}\sim 7 \\
\left(\mathrm{Mpa} \mathrm{m}^{1 / 2}\right)\end{array}$ & {$[20]$} \\
\hline Beryllium & $\begin{array}{c}\text { Temperature: }<435^{\circ} \mathrm{C} \\
\text { Neutron fluence: } 1.5 \times 10^{21} \mathrm{n} / \mathrm{cm}^{2} \\
\text { Neutron energy: }>1 \mathrm{MeV}\end{array}$ & Fracture toughness & $\begin{array}{c}\sim 10 \\
\left(\mathrm{Mpa} \mathrm{m}^{1 / 2}\right)\end{array}$ & {$[20]$} \\
\hline Graphite (N3M) & $\begin{array}{c}\text { Temperature: } 600{ }^{\circ} \mathrm{C} \\
\text { DPA: } 5\end{array}$ & Volume change & $-2 \%$ & [21] \\
\hline Graphite (ATR-2E) & $\begin{array}{c}\text { Temperature: } 500^{\circ} \mathrm{C} \\
\text { DPA: } 21\end{array}$ & Creep strain & $1 \%$ & {$[22]$} \\
\hline
\end{tabular}

TABLE III. Experimental reference values of radiation damage effects in beryllium and graphite. 
different expansion rates which result in generating high stresses within the lattice. The overall volumetric change in graphite depends on grade and inner structure. The strain to failure is reduced in irradiated graphite and the strength and elastic modulus are increased [22]. The rapid increase in strength of irradiated graphite is largely saturated at doses higher than 1 DPA. Above 1 DPA, a more gradual increase in strength occurs because of structural changes within the graphite lattice. As for temperature effects, it has been observed that when high energy neutrons collide with graphite atoms, the DPA rate is flux dependent and independent of the lattice temperature [23].

In beryllium, the large production levels of helium gas as shown in Fig. 6 arise from the ${ }^{9} \mathrm{Be}(\mathrm{n}, 2 \mathrm{n}) 2{ }^{4} \mathrm{He}$ reactions. At temperatures higher than $200{ }^{\circ} \mathrm{C}$, helium bubbles start to form and result in a positive volume change (swelling) [19]. According to the reference values listed in Table III, the effect of helium production levels on volumetric change is temperature dependent. Irradiation also seems to lower the fracture toughness of beryllium at all testing temperatures [20]. The lowest fracture toughness of $\sim 7 \mathrm{Mpa} \mathrm{m}^{1 / 2}$ has been observed at operating temperatures below $230{ }^{\circ} \mathrm{C}$. As for the effect of radiation damage on the thermal conductivity of beryllium, irradiation temperature appears to be a determinant factor as the most rapid irradiation-induced reduction in thermal conductivity was observed to occur at low irradiation temperatures and doses [19]. The reduction in the thermal conductivity of the target structural materials is of particular importance and will need to be considered upon designing cooling systems.

\section{A. DPA}

The DPA values of different target materials operating under conditions similar to that of Target Station 1 in ISIS were estimated using the FLUKA code [24]. FLUKA was

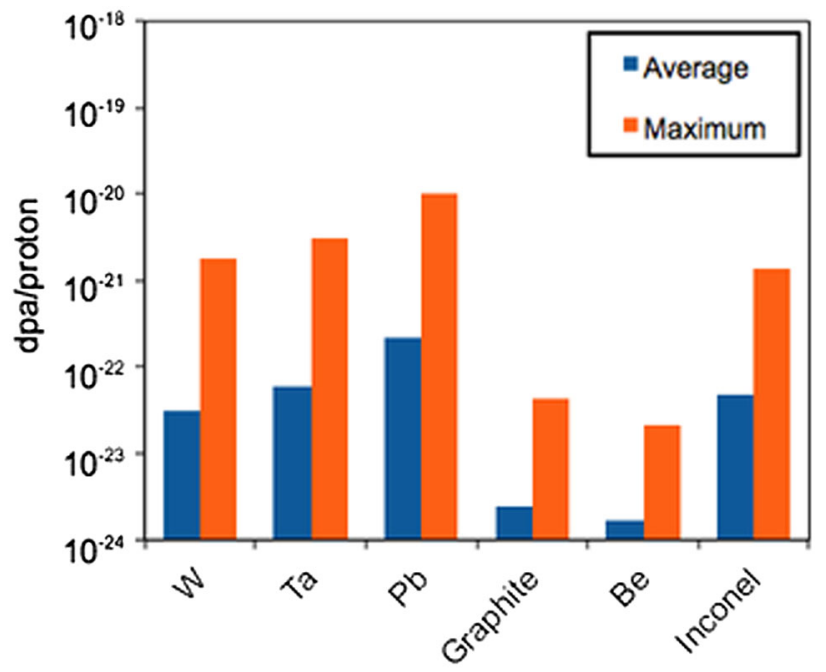

FIG. 4 (color online). DPA values per incident proton of $800 \mathrm{MeV}$ kinetic energy for different target materials.
TABLE IV. Simulation parameters of the radiation damage case.

\begin{tabular}{lcc}
\hline \hline Parameter & Value & Unit \\
\hline Target dimensions & $10 \times 10 \times 36$ & $\mathrm{~cm}$ \\
Beam kinetic energy & 800 & $\mathrm{MeV}$ \\
Beam profile & Gaussian $(\sigma=1.7)$ & $\mathrm{cm}$ \\
Displacement damage threshold & Value $(\mathrm{eV})$ \\
$\mathrm{W}$ & 90 \\
$\mathrm{Ta}$ & 53 \\
$\mathrm{~Pb}$ & 25 \\
Graphite & 30 \\
Be & 31 \\
Inconel & 40 \\
\hline \hline
\end{tabular}

chosen to simulate the DPA and helium production levels in target materials because of its recently added advanced capability of studying radiation damage [25]. Moreover, FLUKA is used for protection studies for the LHC and reported to offer the most realistic predictions of radiation induced damage to materials [25]. The operating conditions that were used to obtain the results shown in Fig. 4 are listed in Table IV. The results in Fig. 4 were normalized by the number of incident protons and therefore can be used to estimate the total DPA for different beam currents and running times.

Two DPA values were calculated for each material: an average value that was derived by dividing the total number of DPAs by the number of volumetric mesh cells in the target, and a maximum value that corresponds to the highest DPA value in a single cell of $1 \mathrm{~cm}^{3}$. Figure 4 clearly shows that lead had the highest average and maximum DPA values, while beryllium had the lowest DPA values. From a material point of view, two major parameters affected the DPA value: the density and the displacement damage threshold, which is defined as the minimum kinetic energy that an atom in a solid needs to be permanently displaced from its lattice site to a defect position. Although tungsten has a higher density than tantalum, it suffered less DPA because its displacement damage threshold is higher. Values of displacement damage threshold of the target materials studied in Fig. 4 are also listed in Table IV.

Figure 5 shows the variations of DPA across the target in the beam direction. Low $\mathrm{Z}$ target materials such as graphite and beryllium have their peak DPA location around $10 \mathrm{~cm}$ behind the impact point and have more uniformly distributed DPA levels across the target. On the other hand, high Z target materials have their DPA peak located only $2-3 \mathrm{~cm}$ behind the impact point, and their DPA level drops significantly in the beam forward direction due to increased electronic energy losses. This highlights another potential advantage of composite and segmented solid targets in reducing high DPA levels. Inconel was not included in this section as it was only proposed as target window material. 


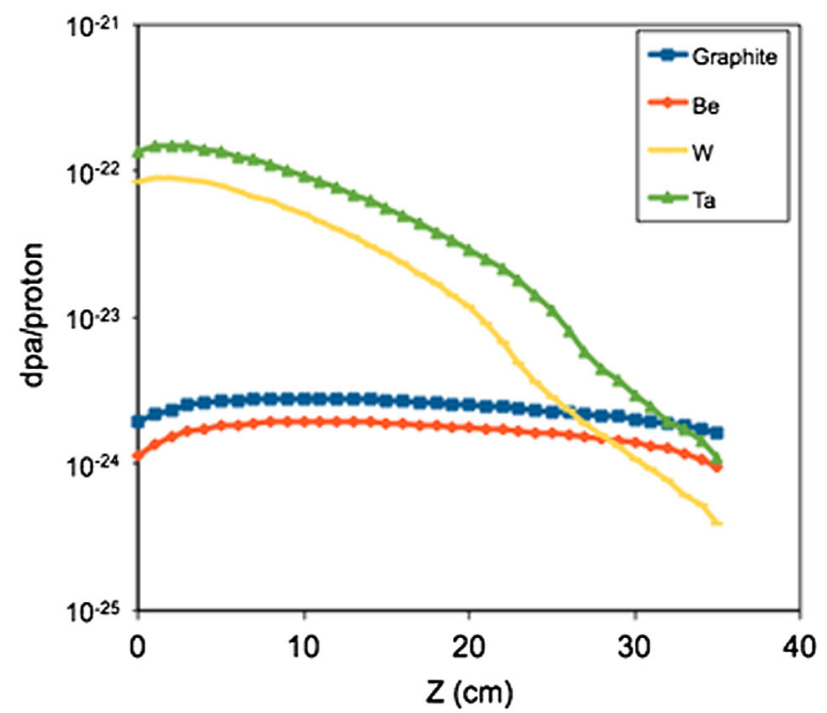

FIG. 5 (color online). DPA variations with target depth for different target materials.

\section{B. Helium production}

The radiation field in a spallation target produces higher levels of light charged particles such as hydrogen and helium than does a typical nuclear reactor. The buildup of these gases, especially helium, can lead to material degradation through embrittlement and swelling in the target and its structural materials - in particular the beam window [26]. Therefore, reliable predictions of helium production are essential for high power target development. Figure 6 shows the total helium (He-3 and $\mathrm{He}-4)$ production levels in different target materials. Both graphite and beryllium have relatively higher production levels than do other materials.

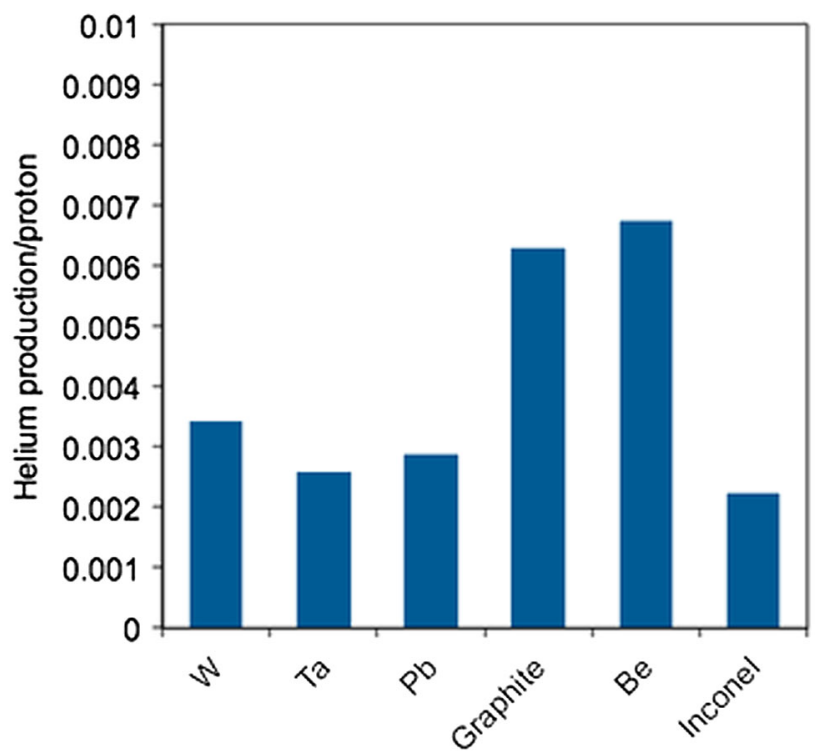

FIG. 6 (color online). Total helium production in different target materials.

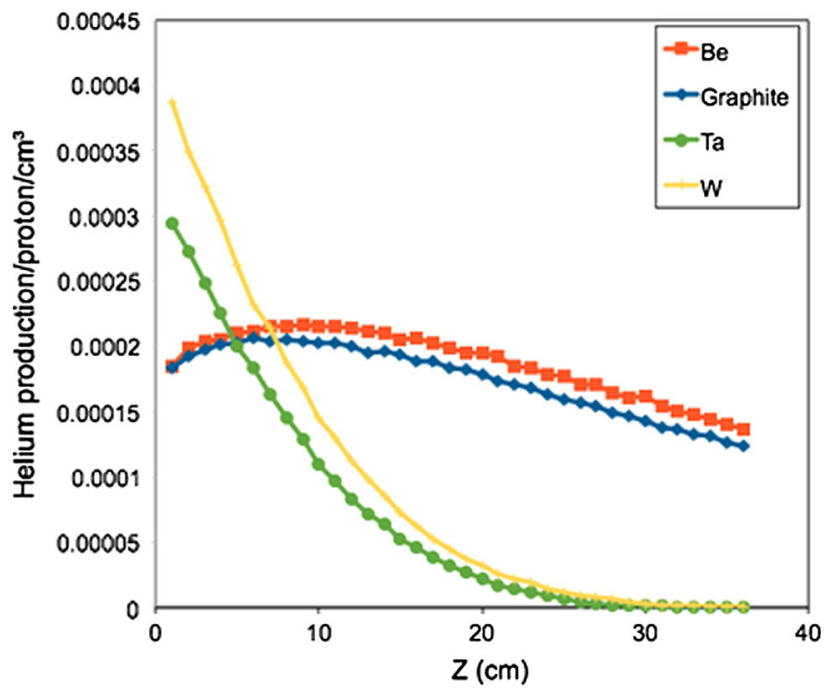

FIG. 7 (color online). Variations of the total helium production with target depth for different materials.

This is due to the added $(\mathrm{n}, \alpha)$ reactions in these two materials. Tantalum shows a helium production level comparable to that of inconel; heavy elements such as tantalum have also been proposed as target window materials [27]. Another factor that may affect the consequences of helium production in target materials is the diffusion coefficient in the irradiated medium. Gas diffusion, which highly depends on the operating temperature, affects the rate and magnitude of voids formation in the target material.

Figure 7 shows variations in the total helium production with target depth for different materials. Although both graphite and beryllium have higher total helium production levels than the other materials, heavy elements such as tungsten and tantalum have higher helium production peak value-approximately double that of beryllium and graphite. Similar to the findings of the DPA analysis, above, heavy elements were found to have their helium production concentrated in the beam impact region, with very low production values at the rear of the target block. Light elements, on the other hand, were found to reach their helium production peak value after $5-10 \mathrm{~cm}$ from the impact point, before production slowly decreased.

\section{CONCLUSION}

In this paper, we studied three major aspects related to the operation and design of high power targets. A figure-ofmerit approach was used to compare the resistance of target materials to thermal stresses as well as the thermohydraulic performance of candidate target coolants. The figure-ofmerit analysis showed that there are some gaps in the materials' data, particularly in regards to tantalum. Low Z target materials, particularly graphite, seem to have a significantly better resistance to thermal stresses due to 
their low density and therefore low volumetric heat deposition. However, beryllium was found to have the highest rate of thermal resistance decrease over the temperature range, where data was available. This was due to a relatively higher coefficient of thermal expansion.

The coolant performance analysis showed that, although water has superior heat transfer efficiency, it absorbs too many neutrons compared to other candidate coolants. Pressurized helium seems to be a good coolant option, as it offers negligible neutron absorption and good thermal properties compared to other gaseous coolants.

On the issue of radiation damage, the total and spatial variations of DPA and helium production were reported for different target materials in a fixed target geometry. Heavy and light elements showed different spatial variations due to the effects of other factors, such as electronic energy losses and the spatial distribution of primary and secondary particles.

[1] P. Hurh, O. Caretta, T. Davenne, C. Densham, P. Loveridge, and N. Simos, Proceedings of 2011 Particle Accelerator Conference (PAC2011), New York, NY, USA.

[2] N. Simos, M. Bishai, and N. V. Mokhov, Nucl. Phys. B, Proc. Suppl. 229, 506 (2012).

[3] F. Carsughi, H. Derz, P. Ferguson, G. Pott, W. Sommer, and H. Ullmaier, J. Nucl. Mater. 264, 78 (1999).

[4] PASI WP1 online page [http://pasi.org.uk/Targets].

[5] F. Sordo, P. T. Leòn, and J. M. Martinez-Val, Nucl. Instrum. Methods Phys. Res., Sect. A 574, 232 (2007).

[6] J. R. J. Bennett, G. P. Skoro, C. Booth, S. J. Brooks, R. A. Brownsword, T. R. Edgecock, C. J. Densham, S. A. Gray, A. J. McFarland, N. Simos, and D. Wilkins, J. Nucl. Mater. 377, 285 (2008).

[7] J. R. J. Bennett, G. P. Skoro, J. Back, S. J. Brooks, T. R. Edgecock, S. A. Gray, A. J. McFarland, K. J. Rodgers, and C. N. Booth, Nucl. Instrum. Methods Phys. Res., Sect. A 646, 1 (2011).

[8] O. Caretta, T. Davenne, P. Loveridge, and C. J. Densham, J. Nucl. Mater. 433, 538 (2013).

[9] J.H.W. Simmons, Radiation Damage in Graphite (Pergamon Press, New York, 1965).
[10] H. Nifenecker, O. Meplan, and S. David, Accelerator Driven Subcritical Reactors (Institute of Physics Publishing, Bristol, England, 2003).

[11] W. Kalbreier, W. C. Middelkoop, and P. Sievers, CERN Lab Technical Report No. II/BT/Int/73-2, CERN, 1973.

[12] D. P. H. Hasselman, Ceramurgia Intl. 4, 147 (1978).

[13] J. W. Davis and P. D. Smith, J. Nucl. Mater. 233-237, 1593 (1996).

[14] T. A. Broome, in Proceedings of the 5th European Particle Accelerator Conference, Sitges, Barcelona, Spain, 1996, p. 267.

[15] G. Hewitt, Introduction to Nuclear Power (Taylor \& Francis Group, London, 1987).

[16] M. B. Chadwick et al., Nucl. Data Sheets 112, 2887 (2011).

[17] J. O. Stiegler and L. K. Mansur, Annu. Rev. Mater. Sci. 9, 405 (1979).

[18] G. R. Longhurst and R. D. Rohe, in Proceedings of the 8th IEA International Workshop on Beryllium Technology (Technical Report No. INL/CON-07-12959, Idaho National Laboratory, 2007).

[19] R. B. Jones, in Proton Accelerators for Science and Innovation 2nd Annual Meeting, RAL-UK, 2013.

[20] R. Chaouadi, F. Moons, and J. L. Puzzolante, at The IEA International Workshop on Beryllium Technology for Fusion, Mito, Japan (Technical Report, Japan Atomic Energy Research, 1998).

[21] T. D. Burchell and W. P. Eatherly, J. Nucl. Mater. 179, 205 (1991).

[22] T. D. Burchell, in Comprehensive Nuclear Materials, edited by R. J. M. Konings (Elsevier, Oxford, 2012), pp. 299-324.

[23] D. G. Schweitzer, Phys. Rev. 128, 556 (1962).

[24] G. Battistoni, F. Cerutti, A. Fassò, A. Ferrari, S. Muraro, J. Ranft, S. Roesler, and P. R. Sala, in Hadronic Shower Simulation Workshop, AIP Conf. Proc. No. 896 (AIP, New York, 2007), pp. 31-49.

[25] A. Fasso, A. Ferrari, G. Smirnov, F. Sommerer, and V. Vlachoudis, Prog. Nucl. Sci. Technol. 2, 769 (2011).

[26] P. Jung, J. Nucl. Mater. 301, 15 (2002).

[27] D. Hilscher, C.-M. Herbach, U. Jahnke, V. Tishchenko, M. Enke, D. Filges, F. Goldenbaum, R.-D. Neef, K. Nnighoff, N. Paul, H. Schaal, G. Sterzenbach, A. Letourneau, A. Bhm, J. Galin, B. Lott, A. Pghaire, and L. Pienkowski, J. Nucl. Mater. 296, 83 (2001). 